Atomistic modeling of the Ge composition dependence of solid phase epitaxial regrowth in SiGe alloys

M. Prieto-Depedro, A. Payet, B. Sklénard, and I. Martin-Bragado

Citation: Journal of Applied Physics 122, 105702 (2017); doi: 10.1063/1.4999987

View online: http://dx.doi.org/10.1063/1.4999987

View Table of Contents: http://aip.scitation.org/toc/jap/122/10

Published by the American Institute of Physics

\section{AP| Journal of Ar Applied Physics}

Save your money for your research.

It's now FREE to publish with us no page, color or publication charges apply.
Publish your research in the

Joumal of Applied Physics

to claim your place in applied

physics history. 


\title{
Atomistic modeling of the Ge composition dependence of solid phase epitaxial regrowth in SiGe alloys
}

\author{
M. Prieto-Depedro, ${ }^{1, a)}$ A. Payet, ${ }^{2,3,4}$ B. Sklénard, ${ }^{2,3}$ and I. Martin-Bragado ${ }^{5, b)}$ \\ ${ }^{1}$ Polytechnic University of Madrid, E.T.S. de Ingenieros de Caminos, 28040 Madrid, Spain \\ ${ }^{2}$ Univ. Grenoble Alpes, Grenoble F-38000, France \\ ${ }^{3}$ CEA, LETI, MINATEC Campus, Grenoble F-38054, France \\ ${ }^{4}$ STMicroelectronic, 850 Rue Jean Monnet, Crolles Cedex 38926, France \\ ${ }^{5}$ UCAM, Universidad Católica de Murcia, Campus de los Jerónimos, 30107 Guadalupe (Murcia), Spain
}

(Received 3 April 2017; accepted 11 August 2017; published online 12 September 2017)

\begin{abstract}
The solid phase epitaxial regrowth (SPER) of SiGe alloys has been studied using atomistic simulation techniques. Molecular Dynamics (MD) simulations reproduce the recrystallization process of amorphous structures created in two different ways: introducing atoms at random positions according to the crystalline density and carefully relaxing the structure; and using a bond switching algorithm by means of ab initio. Activation energies are confronted, and the first method is validated as an efficient way to generate amorphous-crystalline structures suitable to study SPER processes. The MD extracted results show that the SPER rate does not vary monotonically with the Ge composition; instead, activation energies reveal a non-linear behaviour with the addition of Ge, due to the two-part behaviour of the SPER rate: SPER rate itself and a hypothesized extra strain due to the bond length difference. Since SPER is a thermally activated process, nudged elastic band calculations are carried out in order to extend the previous assumption. The energy barrier for an atom to attach to the crystalline phase is computed. The extracted values confirm the presence of the mentioned strain contribution required for an atom to recrystallize when it is not as the same type of the bulk. Published by AIP Publishing. [http://dx.doi.org/10.1063/1.4999987]
\end{abstract}

\section{INTRODUCTION}

Silicon-Germanium (SiGe) alloys are of great interest for semiconductor manufacturing, being used to improve conventional microelectronic device performance. SiGe alloys are currently integrated into pMOSFETs as embedded or raised source/drain materials or channel materials in order to increase the hole mobility. ${ }^{1}$ Solid phase epitaxial regrowth (SPER) is a suitable technique to grow high quality SiGe layers over a silicon substrate. As a consequence of its renewed interest to the industry, modeling the microscopic mechanisms taking place during SPER of SiGe alloys has led to several works. ${ }^{2-6}$

The case of SPER in pure $\mathrm{Si}$ and Ge has been widely studied over a large range of temperatures, described by an Arrhenius expression with activation energies of $2.6-2.7 \mathrm{eV}$ (Refs. 7 and 8) and 2.0-2.2 eV, ${ }^{7,9}$ respectively. Its dependent behaviour on substrate orientation, ${ }^{10,11}$ external strain, ${ }^{12,13}$ and impurities are modeled, ${ }^{14}$ using techniques that range from the atomic to continuum scale. However, the SPER in $\mathrm{SiGe}$ alloys has not been as widely investigated as $\mathrm{Si}$ or Ge. The crystallization kinetics of fully relaxed $\mathrm{Si}_{1-x} \mathrm{Ge}_{x}$ alloy layers have been experimentally studied by Kringhoj and Elliman. ${ }^{15}$ The extracted results revealed a non-linear activation energy profile depending on the germanium composition. For approximately $x=0.2$, the value exceeds the pure Si SPER activation energy. Accepting the bond breaking event to be responsible for the regrowth as described by the

\footnotetext{
${ }^{\text {a)} E l e c t r o n i c ~ m a i l: ~ m . p r i e t o d @ a l u m n o s . u p m . e s ~}$

b)Electronic mail: imartin2@ucam.edu
}

dangling bond model, ${ }^{16,17}$ further confirmed by Aziz, ${ }^{18}$ the activation energy should decrease monotonically from the pure Silicon activation energy to the pure Ge value. Based on this assumption, Payet et $a l^{5}$ presented a comprehensive Lattice Kinetic Monte Carlo (LKMC) model, where the microscopic competition occurring during SPER is also taken into account.

The present work is aimed at modeling the SPER in SiGe alloys by using atomistic simulation techniques. The computational technique used for this purpose is Molecular Dynamics (MD), which has been determined as an adequate tool to simulate recrystallization processes. ${ }^{4,19}$ The amorphous regions used in the performed simulations are obtained by using two different methods: (a) the amorphous region is created following an artificial method based on the amorphous $(\alpha)$-Si density, see Ref. 13, and (b) in order to validate this technique as an efficient and rapid way of generating the amorphous phase, bond defects are introduced into generated structures by means of $a b$ initio. In this frame, the crystallization of fully relaxed $\mathrm{Si}_{1-x} \mathrm{Ge}_{x}$ alloys of different compositions is examined and activation energies depending on the Ge composition are then compared to literature data in terms of SPER rates.

With SPER being a thermally activated process, the Nudged Elastic Band (NEB) method is used in order to compute the energy barrier for an atom to recrystallize in four different cases. On the one hand, for $x=0$, two possible bond types are considered at the interface: $\mathrm{Si}-\mathrm{Si}$ and $\mathrm{Si}-\mathrm{Ge}$, meaning that the two atoms involved in the recrystallization process are $\mathrm{Si}$ and $\mathrm{Ge}$ for a pure $\mathrm{Si}$ configuration. On the other hand, for $x=1, \mathrm{Ge}-\mathrm{Ge}$ and $\mathrm{Ge}-\mathrm{Si}$ bond types are taken into account. 


\section{MOLECULAR DYNAMICS: MODEL AND SET-UP}

MD computes the trajectories of all particles at each time step by solving the Newton equations of motion to evolve the whole system in time. Equations of motion are integrated using the Verlet algorithm using a time step of $2 \mathrm{fs}$. Classical interatomic potentials are used to describe interactions between atoms. In the present study, the Tersoff interatomic potential within its third parametrization is used (T3). ${ }^{20} \mathrm{~T} 3$ accurately accounts for the stability of the crystalline structure and also reproduces the properties of the amorphous phase, giving a reasonable description of $\alpha$-c interfaces for SPER. ${ }^{19,21,22}$ The only drawback is that T3 overestimates the melting point temperature of both $\mathrm{Si}$ and Ge. ${ }^{23}$ However, this limitation is crucial to the observation of SPER using MD, since the temperature has to be high enough to accelerate the system dynamics but below the melting point. This fact leads to carry out MD simulations at temperatures ranging from 1700 to $2000 \mathrm{~K}$.

MD simulations are carried out with a standard MD code, LAMMPS. ${ }^{24}$ Simulations are conducted using the structure shown in Fig. 1; a few atomic planes at the bottom are held fixed in their perfect lattice positions, acting as a substrate. Crystalline-atoms (c-atoms) are next, acting as a seed for the recrystallization, in contact with the $\alpha$-phase. Dimensions of the $\langle 110\rangle$ aligned box-shape in (001) SiGe substrates are $45 \times 100 \times 25\left(\AA^{3}\right)$, containing 5256 atoms, with the lattice parameter obtained according to Dismukes et al. ${ }^{25}$ depending on the Ge composition.

Periodic boundary conditions are applied along $\mathrm{x}$ and $\mathrm{z}$ directions, while the top surface is left free in order to accommodate volume variations. By using a canonical fixed volume dynamics (NVT), the whole system is annealed at $1000 \mathrm{~K}$ for $20 \mathrm{ps}$ before starting the regrowth process, performed at different temperatures ranging from 1750 to $1900 \mathrm{~K}$ every $50 \mathrm{~K}$ until complete recrystallization is reached.

The canonical ensemble NVT (i.e., fixed volume and temperature) is used. The Nose-Hoover thermostat controls

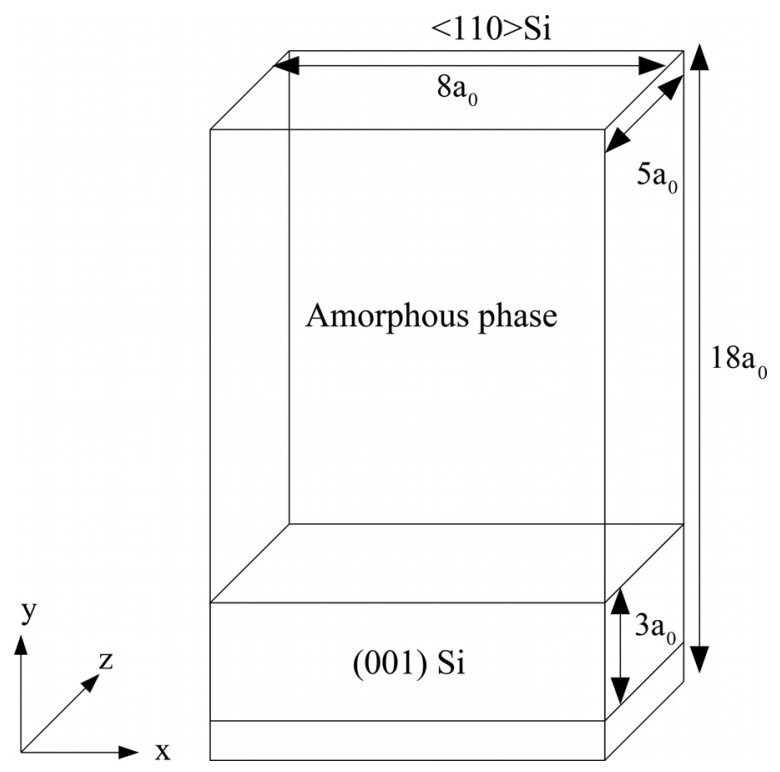

FIG. 1. Schematic configuration of the simulation box. First atomic planes are held fixed acting as a substrate. the temperature during the initialization stage, where the system is annealed at $1000 \mathrm{~K}$ for $20 \mathrm{ps}$. After the equilibration approach, the regrowth process starts by coupling the system to a temperature bath ranging from 1750 to $1900 \mathrm{~K}$ every $50 \mathrm{~K}$ until the complete recrystallization is reached. The velocity is rescaled at each time step with a thermostat time constant of $10 \mathrm{ps}$.

To have an objective criterion to determine the interface position during the thermal annealing, the structural factor is computed as a function of the position in the growth direction $\operatorname{as}^{26}$

$$
S(y)=\frac{1}{N}\left\langle\sum_{j} e^{i \vec{k} \cdot \overrightarrow{r_{j}}}\right\rangle,
$$

where $\vec{k}$ is the reciprocal lattice vector parallel to the $\alpha$-c interface, $r_{j}$ are the atom positions, and $N$ is the total number of atoms in the $j$ region. A value of 1.0 indicates the perfect crystal, 0.0 represents the complete disorder, and 0.5 represents the $\alpha$-c interface. The regrowth rate is calculated from the movement of the $\alpha$-c interface, see Fig. 2 for $11 \%$ of Ge concentration where the linear evolution of the interface with time is evidenced. The MD results for the temperature dependent SPER allow us to obtain from the Arrhenius fitting the activation energy, as shown in Fig. 2 for temperatures ranging from 1750 to $2000 \mathrm{~K}$.

\section{THE AMORPHOUS STRUCTURE}

Amorphous SiGe in the regrowth region is introduced by ion implantation during device manufacturing. Simulating this process should be the best approach to reproduce SPER and be the mimic to the experiment; however, the description of ion implantation by means of MD is not realistic since a

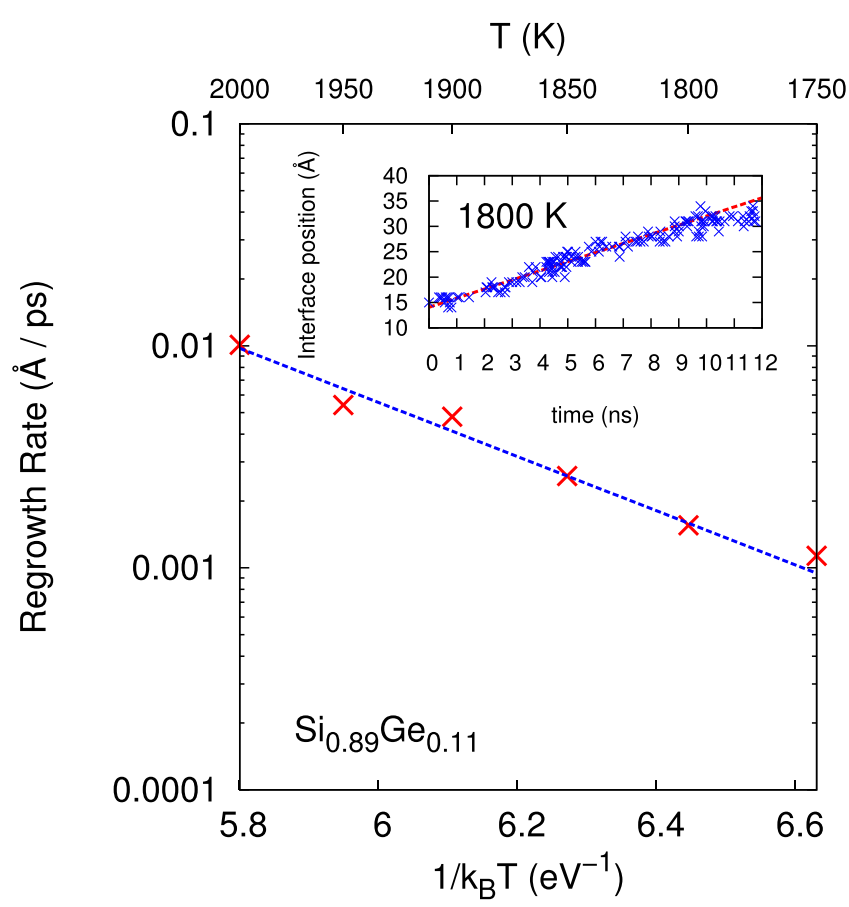

FIG. 2. Arrhenius fitting for $\mathrm{Si}_{0.89} \mathrm{Ge}_{0.11}$ for temperatures ranging from 1750 to $2000 \mathrm{~K}$. Inset: $\alpha$-c interface position versus the annealing time at $1800 \mathrm{~K}$. 

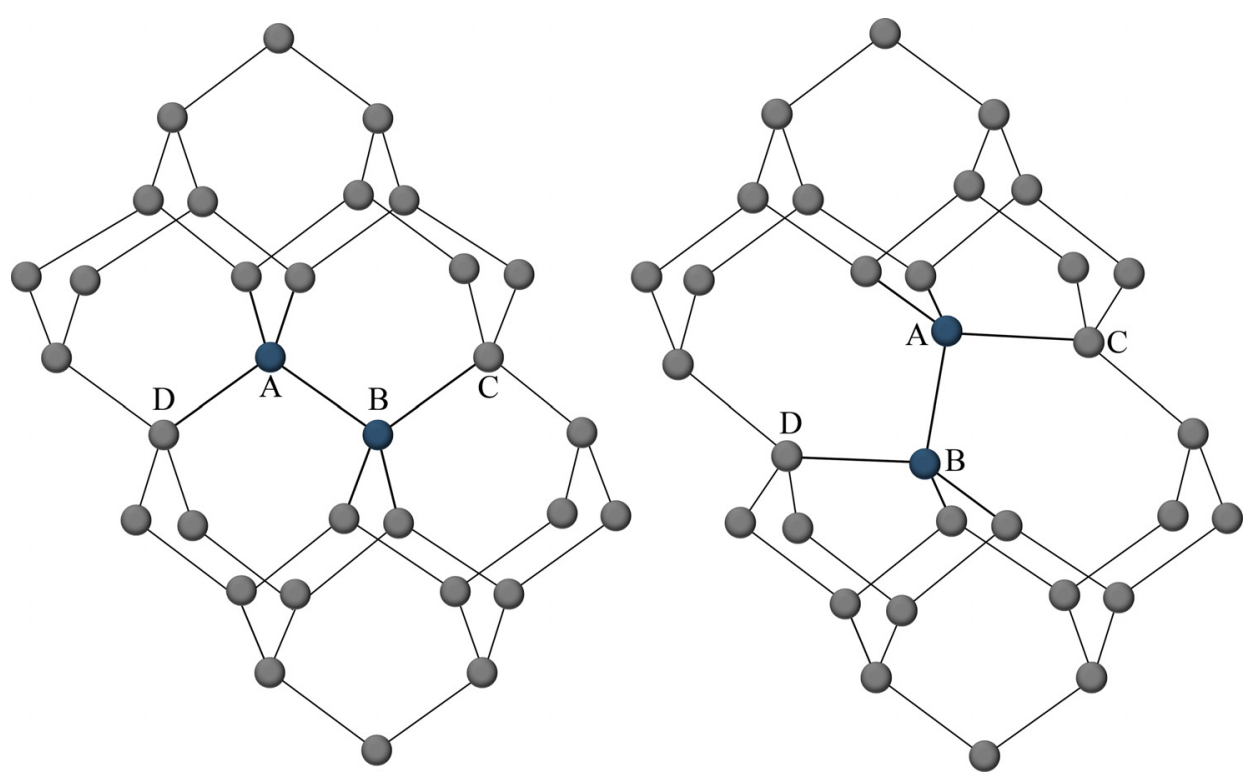

FIG. 3. Structure of the bond defect. ${ }^{33}$ The local distortion of the silicon lattice consists of five-fold and sevenfold membered rings. ${ }^{32}$ high number of implantations would be required to get a good quality sample, being computing-resource and time consuming. Instead, alternative methods are used to create the amorphous volumes, such as a melting-quenching method ${ }^{27}$ or a bond switching algorithm. ${ }^{28}$

The present work presents an alternative and faster method to generate realistic amorphous clusters. This artificial method used to create the computational structure consists of knowing the exact number of c-atoms contained in the region to be amorphized, and these are added to the cell at random positions. As a consequence, atoms are highly overlapped so an energy minimization of the system is performed using the Conjugate Gradient Method (CGM).

Regrowth simulations are then repeated from two to three times with different seeds to specify the initial random positions. Extracted activation energies should converge, and so the final value is an average of all obtained values. This procedure has been used to simulate SPER before with successful results, ${ }^{13}$ and now, it is validated against the generation of the amorphous phase by introducing bond defects.

This second method studies the recrystallization process by using several crystalline samples where selected areas are rendered amorphous by introducing a bond defect by means of $a b$ initio and the subsequent relaxation of the supercell containing 216 atoms. As shown in Fig. 3, the bond defect introduces a local distortion of the lattice creating two five-fold and two seven-fold membered rings packed together, typical of the amorphous state. ${ }^{21}$ The crystalline to amorphous transition under irradiation involves this kind of defect. ${ }^{29,30}$ Indeed, during ion implantation, pairs of interstitial-vacancies are created due to cascades, the so-called IV pairs. Tight-binding MD simulations of Tang et al. ${ }^{31}$ report that under a certain condition, the IV-pair creates a metastable defect structure with an annihilation energy of $1.1 \mathrm{eV}$. Cargnoni et al. ${ }^{32}$ further confirm the energy barrier and also that IV-pair defect and the bond defect are closely related. To create a bond defect, two atoms are displaced. The relaxed positions of these two atoms are determined by using density functional theory (DFT) methods, described within the GGA-PBE functional with a $245 \mathrm{eV}$ cut-off energy, $2 \times 2 \times 2 \mathrm{k}$-point mesh, and $0.01 \mathrm{eV} / \AA$ force tolerance.

The displacement of atoms with respect to their perfect lattice position is tracked. All possible symmetries are taken into account by expressing the displacement vector according to a third non-moving atom, which could be either $\mathrm{C}$ or D, as seen in Fig. 3. To create the amorphous structure, three crystalline neighbour atoms are randomly chosen and two of them are displaced as stated by the displacement vector obtained before. A bond defect is created, and the two atoms that have been displaced are not considered crystalline anymore and cannot be chosen again for displacement. ${ }^{33}$ The bond switching algorithm is repeated until $25 \%$ of the crystalline atoms have been displaced, and the final structure is then fully relaxed.

Figure 4 shows the radial distribution function (RDF) of the amorphous phase constructed for the MD simulations according to these two different methods, where data obtained from the simulations agree well with the RDF deduced by the experiment. ${ }^{34}$ The angular distribution function is contained

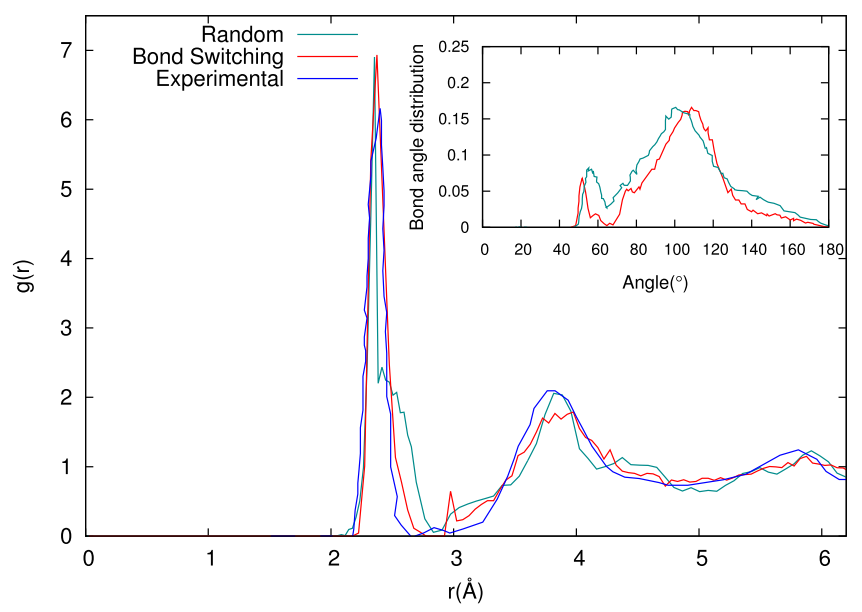

FIG. 4. Radial and angular distribution functions for $\alpha$-Si obtained by the two techniques described on the text. Results of RDF are compared to the experimental data from Ref. 34. 
also in Fig. 4 to further confirm the quality of the amorphous structures.

Extracted activation energies for different Ge compositions obtained by using both methods are compared. As shown in Fig. 5, the maximum does not occur for the same Ge concentration due to statistical variations. There are a few differences regarding the MD simulation conditions, such as the total number of atoms in the supercell. Also for the bondswitching case, the velocities of the non-frozen atoms are initialised with a Gaussian distribution around $300 \mathrm{~K}$, followed by a heat bath of $500 \mathrm{~K}$ for $0.5 \mathrm{~ns}$ to further complete the initialisation. Despite this fact, results contained in Fig. 5, see filled and empty green circles, reveal that the profiles are well matched. So, regrowth velocities obtained in the two cases are very similar. The alternative method has been shown as an efficient way to generate high quality amorphous regions, very suitable to reproduce SPER processes.

\section{NUDGED ELASTIC BAND CALCULATIONS}

Considering SPER as a thermally activated process, the rate for an atom to recrystallize within the Transition State Theory $^{35}$ can be approximated as

$$
\nu=\nu_{0} \exp \left(-\frac{E_{a}}{k_{B} T}\right),
$$

where $\nu_{0}$ is the pre-exponential factor, $E_{a}$ is the activation energy, and $k_{B}$ is the Boltzmann constant. NEB first proposed by Mills et al. ${ }^{36}$ is an efficient and widely used method for calculating reaction pathways of atomic-scale solid-solid transformations through sampling a minimum energy path (MEP). NEB allows us to track the atomic structure evolution by sampling the MEP between the initial and the final

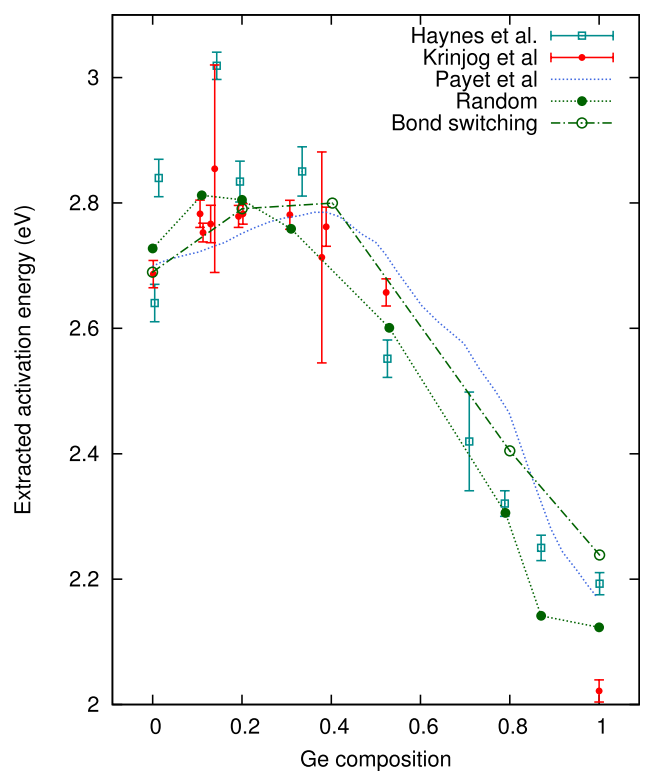

FIG. 5. Activation energy for SPER as a function of Ge concentration. Experimental data from Haynes et al. ${ }^{7}$ are represented as empty squares and Kringhoj and Elliman. ${ }^{15}$ as filled circles with error bars. LKMC model ${ }^{5}$ is presented as a dashed line. MD simulation results are included as filled circles when the $\alpha$-phase is obtained as explained in Sec. II. Results from bond switching algorithm are empty circles. states, both known. The NEB uses N-replicas of the system obtained by linear interpolation using these two mentioned states, connected by harmonic springs to ensure continuity of the path, thus mimicking an elastic band. ${ }^{37}$ The present work addresses the challenge of finding the minimum reaction pathway, i.e., $E_{a}$, for solid phase regrowth transitions. These calculations provide information on the energy of different regrowth pathways in SiGe alloys. Focused on the bonds at the interface, where the bond breaking process is a plausible mechanism for the regrowth, four different cases have been taken into account: Si-Si, Ge-Ge, Si-Ge, and Ge-Si. Each bond type has its own activation energy, which will be estimated along the MEP connecting the initial and final states for the atom-dominated mechanism. Simulations are performed by using the set-up described in Sec. II, re-scaled for a total number of 320 atoms. On the one hand, for $x=0$ and $x=1$, considering a $\mathrm{Si}$ atom forming stable bonds at the interface, the MEP is computed, and so activation energies for $\mathrm{Si}-\mathrm{Si}$ and $\mathrm{Si}-\mathrm{Ge}$ bonds are extracted. On the other hand, this process is repeated for a $\mathrm{Ge}$ atom attaching the crystalline phase for $x=0$ and $x=1$, with bond types involved being Ge-Ge and Ge-Si. It should be pointed out that this is the less favorable situation, and it is considered in order to simulate the limiting conditions during SPER. In $\mathrm{SiGe}$ alloys, recrystallizing atoms as well as their first neighbors could be different, e.g., A Si atom surrounded by $2 \mathrm{Si}$ and 2 Ge atoms, and so on.

\section{RESULTS AND DISCUSSION}

The evolution of the $\alpha$-c interface is tracked during the annealing at different temperatures for each Ge composition. Activation energies are extracted by linear interpolation of the regrowth rates against the inverse of temperature. The results are shown in Fig. 5. The activation energy does not vary monotonically between the values for $\mathrm{Si}$ and $\mathrm{Ge}$; instead, the activation energy goes through a maximum for approximately $x=0.2$. According to the dangling bond model, an amorphous atom attaches to the crystalline phase by the rearrangement of bonds at the interface. Regarding this process of breaking and reconnecting bonds in order to propagate the interface, the bonding $\mathrm{Si}-\mathrm{Ge}$ energy should lie between the values of $\mathrm{Si}-\mathrm{Si}$ and $\mathrm{Ge}-\mathrm{Ge}$, since both $\mathrm{Si}-\mathrm{Ge}$ and $\mathrm{Ge}-\mathrm{Ge}$ bond strengths are lower than that of $\mathrm{Si}-\mathrm{Si}^{38}$ As the dangling formation energy is expected to decrease with the Ge content, the presence of a second component in the activation energy must be considered. This extra term is identified as the defect migration energy at the interface by Kringhoj and Elliman. ${ }^{15}$ However, continuous models do not provide information about the

TABLE I. Recrystallization energy barriers calculated by the NEB method for different configurations at the interface. The slowest configuration is considered as the limiting rate, see highlighted values. Activation energies are in eV.

\begin{tabular}{lcccc}
\hline \hline Bond type & E1 & E2 & E3 & E4 \\
\hline $\mathrm{Si}-\mathrm{Si}$ & 2.370 & 2.443 & 2.600 & $\mathbf{2 . 6 0 6}$ \\
$\mathrm{Si}-\mathrm{Ge}$ & 2.041 & 2.379 & 2.943 & $\mathbf{2 . 9 6 1}$ \\
$\mathrm{Ge}-\mathrm{Ge}$ & 0.722 & 1.074 & 2.000 & $\mathbf{2 . 0 1 0}$ \\
$\mathrm{Ge}-\mathrm{Si}$ & 1.289 & 1.574 & 2.251 & $\mathbf{2 . 9 6 0}$ \\
\hline \hline
\end{tabular}



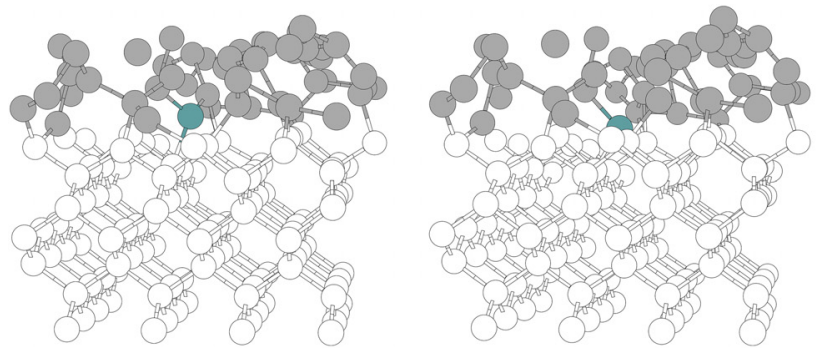

atomic processes taking place during SPER. Payet et al. ${ }^{5}$ introduced their LKMC model, where an intermediate rate is introduced, as first hypothesized by Haynes et al. ${ }^{7}$ The activation energy obtained for $\mathrm{Si}-\mathrm{Ge}$ bonds comes from the linear interpolation of pure $\mathrm{Si}$ and Ge SPER activation energies and a local strain due to the bond length difference in the alloy. This second term explains the maximum.

To confirm the previous point, the NEB method is introduced in order to compute the energy barrier for an atom to recrystallize, as described in Sec. IV. In the process, the involved atom encounters many types of configurations at the interface with their own recrystallization probability. So, several structures have been considered, since SPER is limited to the attachment rate of the slowest crystallizing configuration. The obtained values are summarized in Table I, where considered energy barriers are the highest values, in agreement with the values in Ref. 5. Figure 6 presents the slowest $\mathrm{Si}$ recrystallization configuration extracted from NEB simulations. The MEP is computed for a particular Si atom, identified in green in Fig. 6. The extracted energy barrier to rearrange into the crystalline phase is $2.60 \mathrm{eV}$. This value is in good agreement with the SPER energy in pure Silicon. As shown in Fig. 6, bonds are broken to make topological changes in the structure, and then the loose ones are reconnected. For pure Ge, the calculated energy barrier is $2.01 \mathrm{eV}$, giving a satisfactory result when compared to pure Ge SPER.

Whether the atom to attach is $\mathrm{Ge}$ in $\mathrm{Si}$ or $\mathrm{Si}$ in $\mathrm{Ge}$, the limiting configuration has been found to be different. The presence of $\mathrm{Ge}$ in the $\mathrm{Si}$ lattice, and the opposite, induces local strain due to the differences in bond length, and as a consequence, the energy is found to be higher than the $\mathrm{Si}-\mathrm{Si}$ and $\mathrm{Ge}-\mathrm{Ge}$ bond energies of 2.960 and $2.961 \mathrm{eV}$, respectively.

According to NEB results, it can be confirmed that some of the intermediate configurations require an extra strain for the propagation of the loose bond. This extra strain explains the difference between the bonding formation energy and the SPER activation energy.

\section{CONCLUSIONS}

The Ge composition dependence of SPER activation energies has been investigated by using atomistic simulation techniques. Two different methods of creating the amorphous region have been used. The alternative method proposed in the present work has been evidenced to yield high quality amorphous structures, very suitable to reproduce SPER processes. Using such structures, molecular dynamics simulations have been carried out in order to compute the

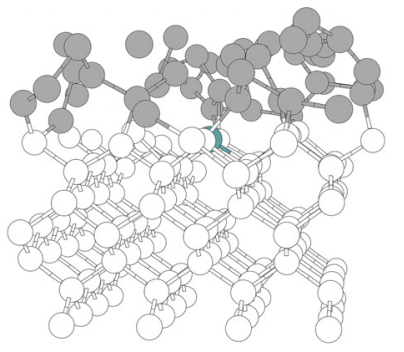

FIG. 6. NEB snapshots for the slowest crystallize configuration in pure silicon. The recrystallized atom is a $\mathrm{Si}$ atom, identified in green. Amorphous atoms are in gray, while crystalline atoms are white. Reconnected bonds show how the atom is rearranged for crystal regrowth to proceed in the $\langle 100\rangle$ direction.

activation energy of a range of strain-relaxed SiGe alloys. The activation energy profile determines the presence of maximum for about $x=0.2$. This profile is in contrast with the dangling bond model where the SPER process is discussed in terms of bond-breaking events.

The present work relies on the assumption that the nonlinear behaviour is due to a two-part behaviour in the SPER rate. The energy needed to attach a new atom into the crystalline phase consists of the SPER energy and an extra term due to the elastic work performed in straining the alloy to overcome bond length. To prove the conclusions obtained by the LKMC model in Ref. 5, where the local strain due to the bond length difference is postulated as this second term, NEB calculations are used to compute the energy barrier in four different cases. Extracted values are in good agreement with SPER activation energies for pure Si and pure Ge configurations. When the atom to rearrange is not as the same type as the bulk, which means that the recrystallized atom is $\mathrm{Ge}$ in pure $\mathrm{Si}$ and $\mathrm{Si}$ in pure $\mathrm{Ge}$, values have been found to be higher than the bonding energies in $\mathrm{Si}-\mathrm{Si}$ and $\mathrm{Ge}-\mathrm{Ge}$. The subsequent results are explained in terms of the mentioned strain required to propagate the crystalline phase when Ge and $\mathrm{Si}$ induce a mismatch in the configuration.

${ }^{1}$ S. Thompson, M. Armstrong, C. Auth, S. Cea, R. Chau, G. Glass et al., "A logic nanotechnology featuring strained-silicon," IEEE Electron Device Lett. 25, 191 (2004).

${ }^{2}$ B. Seiss and D. Dutartre, "Morphology evolution of epitaxial SiGe and Si patterns," Solid-State Electron. 83, 18-24 (2013).

${ }^{3}$ A. Rodríguez, T. Rodríguez, and A. Sanz-Hervás, "Strain compensation by heavy boron doping in SiGe layers grown by solid phase epitaxy," J. Mater. Res. 12, 1698 (1997).

${ }^{4}$ M. Posselt and A. Gabriel, "Atomistic simulation of amorphous germanium and its solid phase epitaxial recrystallization," Phys. Rev. B 80, 045202 (2009).

${ }^{5}$ A. Payet, B. Sklenard, J. Barbé, P. Batude, C. Licitra, A. Papon, J. Hartmann, R. Gonella, P. Gergaud, and I. Martin-Bragado, "Atomistic predictions of substrate orientation impact during SiGe alloys solid phase epitaxial regrowth," in Proceedings of 2016 International Conference on Simulation of Semiconductor Processes and Devices, SISPAD (2016).

${ }^{6}$ A. Payet, B. Sklenard, J. Barbé, P. Batude, C. Tavernier, P. Gergaud, and I. Martin-Bragado, "An atomistic investigation of the composition dependence in SiGe alloys during solid phase epitaxial regrowth," Acta Mater. 106, 290-294 (2016).

${ }^{7}$ T. Haynes, M. Antonell, C. A. Lee, and K. Jones, "Composition dependence of solid phase epitaxy in silicon-germanium alloys: Experiment and theory," Phys. Rev. B 51, 7762 (1995).

${ }^{8}$ R. Elliman, W. Wong, and P. Kringhoj, in Cristallization and Related Phenomena in Amorphous Materials, edited by M. Libera, T. Haynes, P. Cebe, and J. E. Dickinson (MRS Symposia Proceedings, 1994), Vol. 321.

${ }^{9}$ L. Csepregi, R. P. Küllen, J. W. Mayer, and T. W. Sigmon, "Regrowth kinetics of amorphous Ge layers created by $74 \mathrm{Ge}$ and $28 \mathrm{Si}$ implantation of Ge crystals," Solid State Commun. 21, 1019 (1977). 
${ }^{10}$ L. Csepregi, E. F. Kenedy, J. W. Mayer, and T. W. Sigmon, "Substrate orientation dependence of the epitaxial regrowth rate from Si-implanted amorphous Si," J. App. Phys. 49, 3906 (1978).

${ }^{11}$ I. Martin-Bragado, "Importance of twin defect formation created by solid-phase epitaxy growth: An atomistic study," Scr. Mater. 66, 186 (2012).

${ }^{12}$ B. Sklenard, J. Barbé, P. Batude, P. Rivallin, S. Cristoloveanu, and I. Martin-Bragado, "An atomistic investigation of the impact of in-plane uniaxial stress during solid phase epitaxial regrowth," App. Phys. Lett. 102, 151907 (2013).

${ }^{13}$ M. Prieto-Depedro, I. Romero, and I. Martin-Bragado, "Multiscale modeling of defect formation during solid-phase epitaxial regrowth of silicon," Acta Mater. 82, 115-122 (2015).

${ }^{14}$ B. C. Johnson and J. C. McCallum, "Dopant-enhanced solid-phase epitaxy in buried amorphous silicon layers," Phys. Rev. B 76, 045216 (2007).

${ }^{15} \mathrm{P}$. Kringhoj and R. Elliman, "Solid-phase epitaxial crystallization of strain-relaxed $\mathrm{Si}_{1-\mathrm{x}} \mathrm{Ge}_{\mathrm{x}}$ alloy layers," Phys. Rev. Lett. 73, 858 (1994).

${ }^{16} \mathrm{~F}$. Spaepen, "A structural model for the interface between amorphous and crystalline Si or Ge," Acta Mater. 26, 1167 (1978).

${ }^{17}$ F. Spaepen and D. Turnbull, "Kinetics of motion of crystal-melt interfaces," AIP. Conf. Proc. 50, 73-83 (1979).

${ }^{18}$ M. Aziz, "The mechanism of solid phase epitaxy," in Crucial Issues in Semiconductor Materials and Processing Technologies, NATO ASI Series Vol. 222, edited by C. Soffa, F. Priolo, E. Rimini, and J. Poate (Springer, 1992).

${ }^{19}$ E. Lampin and C. Krzeminski, "Molecular dynamics simulations of the solid phase epitaxy of Si: Growth mechanism and orientation defects," J. App. Phys. 106, 063519 (2009).

${ }^{20}$ J. Tersoff, "Modeling solid-state chemistry: Interatomic potentials for multicomponent systems," Phys. Rev. B 39, 5566 (1989).

${ }^{21}$ L. Marqués, L. Pelaz, I. Santos, P. López, and R. Duffy, "Molecular dynamics simulations of the regrowth of nanometric multigate Si devices," J. Appl. Phys. 111, 034302 (2012).

${ }^{22}$ S. Munetoh, K. Moriguchi, A. Shintani, K. Nishihira, and T. Mootola, "Molecular dynamics simulations of solid-phase epitaxy of Si: Defect formation process," Phys. Rev. B 64, 193314 (2001).
${ }^{23}$ S. J. Cook and P. Clancy, "Comparison of semi-empirical potential functions for silicon and germanium," Phys. Rev. B 47, 7686 (1993).

${ }^{24} \mathrm{See}$ http//lammps.sandia.gov for molecular dynamics open-source code.

${ }^{25}$ J. P. Dismukes, L. Ekstrom, and R. J. Paff, "Lattice parameter and density in germanium-silicon alloys," J. Phys. Chem. 68, 3021-3027 (1964).

${ }^{26}$ A. Mattoni and L. Colombo, "Boron ripening during solid-phase epitaxy of amorphous silicon,” Phys. Rev. B 69, 045204 (2004).

${ }^{27}$ W. D. Luedtke and U. Landman, "Preparation and melting of amorphous silicon by molecular-dynamics simulations," Phys. Rev. B 37, 4656 (1988).

${ }^{28} \mathrm{~F}$. Wooten, K. Winer, and D. Weaire, "Computer generation of structural models of amorphous Si and Ge," Phys. Rev. Lett. 54, 1392 (1985).

${ }^{29}$ L. A. Marqués, L. Pelaz, M. Aboy, L. Enríquez, and J. Barbolla, "Microscopic description of the irradiation-induced amorphization in silicon," Phys. Rev. Lett. 91, 135504 (2003)

${ }^{30}$ T. Motooka, "Model for amorphization processes in ion-implanted Si," Phys. Rev. B 49, 16367 (1994).

${ }^{31}$ P. J. P. Tang, M. J. Pullin, and C. C. Philips, "Binding energy of the free exciton in indium arsenide," Phys. Rev. B 55, 4376 (1997).

${ }^{32}$ F. Cargnoni, C. Gatti, and L. Colombo, "Formation and annihilation of a bond defect in silicon: An ab initio quantum-mechanical characterization," Phys. Rev. B 57, 170 (1998).

${ }^{33}$ A. Payet, "Physical modelling of junction fabrication processes on FDSOI substrate for $10 \mathrm{~nm}$ node and below," Ph.D. thesis (University of Grenoble Alpes, 2017).

${ }^{34}$ K. Laaziri, S. Kycia, S. Roorda, M. Chicoine, and J. Robertson, "High resolution radial distribution function of pure amorphous silicon," Phys. Rev. Lett. 82, 3460 (1999).

${ }^{35}$ K. J. Laidler and M. C. King, "Development transition state theory," J. Phys. Chem. 87, 2657-2664 (1983).

${ }^{36}$ G. Mills, H. Jonsson, and G. K. Schenter, "Reversible work transition state theory: Application to dissociative adsorption of hydrogen," Surf. Sci. 324, 305-377 (1995).

${ }^{37} \mathrm{H}$. Espinosa and G. Bao, Nano and Cell Mechanics: Fundamentals and Frontiers (John Wiley and Sons, 2013).

${ }^{38}$ L. Pauling, in Nature of the Chemical Bond, edited by N. Y. Ithaca (Cornell University Press, 1960). 